Zu dem erkalteten Eisessig, der sich nicht gefärbt hat, giebt man nun ein abgekühltes Gemenge gleicher Volumina concentrirter Schwefelsäure und Salpetersäure. Ungefähr 1 Gramm des Säuregemenges wird zu 2 bis $3 \mathrm{Gr}$. des Oeles gesetzt und durch Neigen des Probirrohres allmälig gemischt. Bei Anwendung von Arachisöl entsteht hierbei keine Färbung; wird der Versuch aber mit Sesamöl angestellt, so nimmt der Eisessig sofort eine ziemlich beständige schön gelbe, etwas ins Grüne spielende Farbe an. Man sieht, es handelt sich hierbei um eine Spur eines vielleicht harzartigen Stoffes, welcher im Sesamöl, nicht aber in den andern Oelen, namentlich nicht in dem der Erdnuss enthalten ist. Auch vermittelst Weingeistes kann dieser Körper dem Sesamöl entzogen werden; nach dem Verjagen des Weingeistes färbt sich der geringe Rückstand bei vorsichtigem Zusatze des erwähnten Säuregemisches anfangs bla $\mathrm{u}$, dann grünlich gelb.

\title{
Flora und Fauna des Soolgrabens zu Artern.
}

Von L. Sondermann, Apotheker in Artern.

Das grosse Becken, welches zwischen dem Harz und dem Thüringer Walde liegt, und zwar in der Nähe einer Hebung, welche sich vom Kyffhäuser über Bottendorf nach Wendelstein an der Unstrut in der allgemeinen Richtung von Nordwest nach Südwest verfolgen lässt, enthält die Saline Artern. Nördlich von der Stadt gehen die ältern Glieder der bunten Sandstein-Formation zu Tage aus, und hier findet sich eine Einsenkung, welche mit Gypsschlotten und Erdfällen zusammen $\mathrm{zu}$ hängen scheint.

Vermuthlich aus solchen Schlotten trat in dem sogenannten Salzthale einige tausend Fuss von der Stadt entfernt, umgeben von Gypsmassen, die hier in Kuppen zu Tage treten, die Salzquelle hervor, die früher gradirt und so con- 
centrirt, zur Salzfabrikation diente, jetzt aber wegen störker, erbohrte Soole, als unbrauchbar in die Unstrut läuft.

Im Jahre 1580 kaufte der Kurfürst August von Sachsen von dem Dr. Candler und dem Kramer v. Claus burg die Saline für 40,000 Gulden. Zwei Jahre darauf wurde das jetzige Salzthal mit einer Mauer umgeben, mit Pfannen versehen und in Betrieb gesetzt. Doch wurde bereits im Jahre 1585 das Werk an die Grafen von Schwarzburg und die Pfännerschaft zu Frankenhausen veräussert, welche eine Concurrenz für die Bchwarzbargische Saline Frankenhansen fürchtete, den Betrieb mit der Zeit aussetzte, doch Soolquelle und Salzthal als Eigenthum des Fürsten von Schwarzburg bis in die neueste Zeit behielt.

Erst durch den Staatovertrag von 1816 fiel sie dem Preussischen Staate zu, welcher auch das Salzthal für den Preis von 2000 Thaler acquirirte.

Die Soolquelle selbst liegt in der Mitte des Salzthales, und das Nivean, bis zu welchem die Quelle im Bassin aufsteigt, liegt 411' über dem mittlern Spiegel der Ostsee, die constante Temperatur der Quelle beträgt $13^{0}, 5$ C., die Ausflussmenge dagegen ist verschieden, im Durchschnitt 127,5 C. F. in der Minute, varïrt dagegen von 101 bis 360 C.F. Fluthen und Gewitterregen verstärken die Ausflussmenge, zugleich vergrössert sich dann merkwürdigerweise der Salzgehalt, der im Mittel 3,7\% beträgt, und ein Zeichen ist, dass sie mit Trgewässern in Verbindung steht. Die Zugänge selbst vermuthet man in der Gegend von dem circa 4 Stunden entfernten Agnesdorf, da nach einem dort niedergegangenen Wolkenbruch sich nach kurzer Zeit die Quelle ungewöhnlich ergiebig zeigte. Ein ferneres Zeichen für den Zugang der Tagewässer ist, dass sie hin und wieder organische Stoffe, wie Holz, Wurzeln, Knochen ausstösst, welche erst von oben hinab geschlemmt sein müssen. Durch Bohrversuche ist ermittelt worden, dass die unterliegenden Schichten bestehen aus: 
$335^{\prime}$ aufgeschwemmten Gebirge und Geröllschichten mit Sand und Thon,

$250^{\prime}$ Formation des bunten Sandsteins, 165' Gyps, als tiefste Schicht des bunten Sandsteins,

34' Kalkstein zur Zechstein - Formation gehörend, $173^{\prime}$ Gyps, als Zechstein-Gyps anzusehen.

$957^{\prime}$

Die Soole selbst besteht in 100 Theilen aus:

\begin{tabular}{lr} 
kohlensaurem Kalk & 0,005 \\
kohlensaurem Eisenoxydul & 0,003 \\
Gyps & 0,429 \\
schwefelsaurem Kali & 0,053 \\
Chlormagnesium & 0,061 \\
Chlornatrium & 2,449 \\
Wasser & 97,000 \\
\hline & $10 \overline{0,000 .}$
\end{tabular}

Das ohngefähr 100 Quadratfuss grosse in Bohlen gefasste Bassin hat 3 Quellen, die ziemlich dicht beisammen liegen, und wird von hier aus durch ein gemauertes Gerinne $100^{\prime}$ durch das Salzthal gefuhrt, dort aber, wo die Soole das offene Thor passirt und in das offene Feld tritt, fliesst sie auf thoniger Unterlage nach der Stadt $z u$ in die Unstrut. Ueberrascht ist der Sammler durch die Fülle der Vegetation, die ihm hier entgegentritt und die beiden Ufer des Baches umsäumt. Die durch ibren rigiden Habitus sich unterscheidenden Salzpllanzen wechseln in bunter Reihe mit denen des Thales, die auch auf salzhaltigem Boden kräftig gedeihen.

Grosse Massen von Algen schwimmen vorüber, die an manchen Stellen vom Röhricht gehalten den Bach völlig bedecken.

Doch die Zeit ist günstig, und da wir uns vor keinem Flurschützen zu fürchten brauchen, beginnen wir sofort mit der Einsammlung der Phanerogamen und sammeln auf dem terrassenförmigen Terrain folgende Salzpflanzen.

Am Boden des Baches fluthet mit der Strömung die Ruppia unaritima L. und Ruppia rostellata Koch, während die Ufer bedeckt sind von Spergula media Garcke, Spergula marina Grck., Melilotus dentatus Pers., Lotus corniculatus $\beta$ terni- 
folius NEsb., Bupleurum tenuissinum L., Aster Tripolinm L., Artemisia rupestris L., Artemisia maritima a) salina Wild. b) gallica Wild., Samolus Valerandi L., Glaux maritima L., Plantago maritima L., Chenopodium maritimum Mq., Salicornia herbacea L., Obione pedunculata Mq., Atriplex nitens NEsb., Atriplex maritima Deth., Atriplex litoralis L., A. laciniatum L., Rumex maritimus L., Triglochin maritimum L., Potamegeton marinus $L$., Juncus Gerardi Loisl., Juncus compressus Jacq., Scirpus maritimus L., Carex vulpina L.

Ansserdem haben wir noch $z \mathrm{n}$ sammeln Gelegenheit von:

Rànunculus acris L., Sisymbrium officinale Jacq., Alyssum calycinum L., Cochlearia Armoracia L. , Thlaspi arvensis L., Lepidium ruderale L., Dianthus Carthusianorum L., Holosteum unbellatum L., Myosurus minimas L., Stellaria media Vill, Linum catharticum L., Malva sylvestris L., Althaea officinalis L., Lavatera thuringiaca L., Geranium pusillum L., Erodium cicutarium Ltr., Tetragonolobus siliquosus Rchb., Potentilla reptans, verna, opaca L., Sedum acre L., Eryngium campestre L., Dipsacus sylvestris L., Artemisia campestris L., Centaurea Jacea L., Tragopogon major Jacq., Trag. pratense L., Crepis biennis L., Hieracium Pilosella L., Asperugo procumbens L., Veronica verna L., Ver. praecox All, Salvia pratensis L., Chenopodium rubrum L., Triglochin palustre L., Avena tenuis Mch., Poa annua L., Poa compressa L., Festuca ovina, c) duriuscula d) glauca Sch., Lolium perenne L.; auch Lemna gibba L. finden wir im Brunnen nebenan.

Von den Laubmoosen haben wir Gelegenheit am Ufer und in unmittelbarer Nähe zu sammeln:

Phascum cuspidatum Salb., Phasc. nervicollum Hdw., Barbula ruralis H., und steril Barbula muralis Tim., Barbula rigida Sch., Grimmia pulvinata Smith, Bryum cespitosum L., Fumaria hygrometrica L., Pottia Heimii Turh., Pottia cavifolia Ehrh., Ephemerum cohaerens Ny., Fiedleria subsessilis Rab., Didymodon cordatus Schp. u. Did., luridus Schp., Racomitrium canescens Brid., Orthotrichum obtusifolium Schrad., u. Orth. affine Schd. auf den Pappeln. am Brunnen. Thuidium abietinum B. u. Sch., Thuidium tamariscinum B. u 
Sch., Homalothecium sericeum Sch., Brachythecium rutabulum B. u. Sch., B. salebrosum Schp.:

Von Lebermoosen finden wir Lophocolea minor N. u. Rc.

Von den Flechten:

Parmelia parietina Ach., Parm. pulchella Eh., Baeomyces roseus Pers., Cladonia rufa $\mathrm{Ny}$.

Von den höheren Thieren kommt das kleine Wieselchen Mustela vulgaris L. häufig vor, das neben Eidechsen Lacerta agilis $L$. in Löchern, welehe sich in dem Ufer, das sich terrassenförmig nach dem Gottesacker zu erstreckt, wohnt.

In der Soole tummeln sich Heerden kleiner Fische und zwar 2 Arten Gasterosteus trachurus C. und Gasterost. laevis C., die, sobald man sich ihnen nähert, schnell in den am Boden wuchernden Ruppien sich zu verstecken suchen. Interessant sind die Nester, die sie aus Holzstückchen und Pflanzenresten am Ufer im Schlamme zu bauen verstehen, in welchen sie laichen und sich gegen Feinde vertheidigen.

Einer eigenthümlichen Krankheit sind die Fischchen unterworfen. Einzelne schwellen blasenförmig in der Nähe des Hinterleibes auf, wodurch das Schwimmen verhindert wird, und sie auf die Seite oder den Rücken zu liegen kommen und so in den Algen mit ihren Stacheln hängen bleiben und sterben. Tagelang kann man sie so beobachten, bevor der Tod erfolgt, die Krankheit entsteht durch Ansammlung von Luft, die den Unterleib blasenförmig auftreibt.

Hin und wieder findet man auf diesen Fischen einen niedlichen Schmarotzerkrebs, der grosse Aehnlichkeit mit dem Argutus foliaceus hat, und sich nur durch die beiden anders geformten Saugnäpfe, worin das erste Fusspaar endet, von ihm unterscheidet. Dieser Krebs bewegt sich schnell über die Haut der Fische bin, doch ist derselbe seiner Saugnäpfe wegen nur schwer zu trennen.

Von den Fröschen findet sich in der Nähe des Ufers häufig Rana temporaria oxyrrhinus $L$. vor. Wirft man denselben in die vorüberfliessende Soole, so giebt er einen eigenthümlichen Laut von sich und strebt mit aller Macht das 
Ufer zu gewinnen, ein Zeichen wie höchst unangenehm das Salzwasser auf ihn einwirkt.

Die Orthopteren sind neben dem gemeinen Gryllus campestris durch Gryllotalpa vulgaris Latr. vertreten, deren Röhren man häufig im feuchten Ufer antrifft, so wie eine $10^{\prime \prime \prime}$ lange braune Forficula.

Von den Lepidopteren habe ich bis jetzt wenig beobachtet. Auf der Artemisia gallica trifft man im Sommer hin und wieder eine Raupe mit perlweisser Grundfarbe, funf gelben Streifen, dazwischen mit schwarzen Linien und Punkten. Ob aus den gesammelten Raupen die Cucullia tanaceti, mit der sie der Beschreibung nach übereinstimmt, entstehen wird, muss das Frühjahr lehren. Von den Dipteren findet man im Monat August zwischen Algen umherschwimmend, eine ein bis zwei Zoll lange Larve, der Gattung Cristalis angehörend, in ungeheuren Mengen an, doch da ich kein Fliegenkenner bin, ist die sich daraus entwickelnde Fliege nicht gesammelt und bestimmt worden. Dasselbe gilt auch ron den besonders reich vertretenen Spinnen und Wanzen.

Von den Kerbthieren findet sich ausser dem Schmarotzerkrebs noch massenweise ein Oniscus unter Steinen vor.

Von den Conchilien trifft man im Sommer die niedliche Pupa muscorum L. unter Steinen am Ufer an, während Planorbis spirorbis Müll. im Brunnen nebenan aufzufinden ist.

Gehen wir nun zu den Coleopteren über und fangen zu sammeln an. Hier am Ufer auf bewachsenem Untergrunde liegt eine Partie Steine, von denen wir einige umlegen. Welches Leben - wir haben nicht Hände genug, schnell Alles das zu sammeln, was durcheinander umherläuft. Hier ein Nest rother Ameisen, dazwischen der Claviger longicornis Müll., der blind, von den Ameisen sorgfältig gepflegt und gefuittert wird, dort Dutzende von Dichirotrichus (Bradycellus) pubescens Paj., die zusammen hocken, dazwischen Stenolophus elegans Dej., der sich eiligst aus dem Staube machen will.

Wir heben nun die bei der Reinigung herausgezogenen deckenbildenden Algen und Conferven auf, wo wir jedenfalls reiche Beute machen werden. Richtig! - Wie das von 
Insecten wimmelt und krabbelt - Käfer, Fliegen, Wanzen, Spinnen aller Art laufen bestürzt durch einander, und suchen sich eiligst in ihren selbstgegrabenen Löchern zu verkriechen. Ein Cephalotes vulgaris, in seiner Höhle von den vielen Asseln und Wanzen, die ihm bei der Fülle der Insectenwelt leicht zur Beute fielen, ordentlich fett geworden, daneben eine auf dem Rücken liegende und sich todt stellende Amana convexiuscula March., die wir leicht an der rothbraunen Brust und dito Füssen erkennen, - fürwahr kein schlechter Anfang. -

Schnell eilen die leichtfiissigen kleinen Bembidien, das schwarze metallisch glänzende B. pusillum Gyll,, das gelbe scutellare Dej., und das bronceschimmernde mit dem in der Sonne leuchtenden rothen Fleck am Hintertheile, aspericolle Germ. vorüber - daher hurtig zugegriffen! und bringen sie, um sie nicht $z u$ beschädigen, mit dem befeuchteten Finger in unsere Benzin enthaltende Flïschchen. Dies thun wir auch mit der kleinen Bryaxis Helferi Schrd. und dem kleinen Anthicus humilis Germ., der hier zuweilen vorkommt, sonst aber sicher an den Wurzeln der Salicornia herbacea aufgefunden wird. Auch die drei Dyschirien, D. chalceus Ev., D. extensus Putz und salinus Schaum., die sämmtlich hier zu Hause sind, finden wir, so wie den von Redtenbacher am Neusiedler-See entdeckten Dichirotrichus lacustris Redt. *) der bis jetzt in Deutschland noch nicht aufgefunden worden ist.

Eben daselbst vorsteckt finden wir den sonst so seltenen $5^{\text {IJs }}$ langen metallisch grünglänzenden Anisodactylus

*) Die meisten entomologischen Werke haben den D. lacustris noch nicht aufgenommen, ich füge daher die Beschrcibung nach Dr. Bose bei. Bradycellus lacustris unterscheidet sich leicht von pubescens durch das hinten breitere Halsschild, dasselbe ist bei letzterem mehr herzförmig, vor den fast spitzwinkeligen Hinterecken etwas ausgeschweift. Es ist bei ersterem dichter punktirt, - die Flügeldecken sind bei erster schwächer gestreift, in den Strcifen kaum punktirt, ihre Zwischenräume fein und sehr dicht punktirt, - bei pubescens sind die Streifen der FlügeJdecken wenn auch schwach, doch disht punktirt, ihre $Z$ wischenräume mit einer oder zwei Reihen ziemlich grober Punkte. 
pseudo-aeneus Dej., der eiligst in seinem senkrechten Loche nach der Tiefe geht.

Unter der dichten Decke der Artemisien und Chenopodien, die keinen Sonnenstrahl durchlassen, finden wir auf dem feuchten Boden am Ufer die reich vertretenen Staphylinideen - Homalota meridionalis Muls., Philanthus salinus Kiesw, so wie noch eine Menge dieser schwer zu bestimmenden Familie, während wir auf den sterilen Stellen, die nur hin und wieder von der Salicornia herbacea eingenommen werden, die jagende Cincidela maritima Dej. und auf Plantago und Triglochin maritimum die Chrysomela concinna Steph. und Malachius spinosus Er. antreffen.

Wir bemerken kleine Häufchen Erde, die truppweise auf dem Boden aufgeworfen sind, - sollten hier in dem feuchten Untergrunde Erdwespen wohnen? Wir graben nach und finden perpendiculäre Gänge, die sich 6 bis 10 Zoll tief erstrecken, in denen die seltensten Troglodyten: der Bledius taurus Germ., unicornis Ger., bicornis Ger. und tricornis Hbst. auf - und abkriechen. Finden wir aber die Gänge leer, so gelingt es uns vielleicht in der Abendstunde, zu welcher sie aus ihren Löchern hervorkommen, sie im Fluge zu fangen.

Gehen wir im Frühjahre nach der Soole und ziehen die am Boden wachsenden Ruppien, so wie die obenschwimmenden Algen mit einem Stocke heraus. Wir breiten sie in dïnnen Schichten auf dem Boden aus, und untersuchen sorgfältigst. Hier ist eine Fundgrube der seltensten Sachen. Hydroporus lautus Schaum., Hydrop. parallelogrammus Ahr., Hyd. nigrida Gyll., Philhydrus testaceus, Halypeus fulvicollis Er., Ochthebius foveolatus Germ., Ochth. marinus Payk., sowie ein 'noch unbeschriebenes Philhydrus habe ich hier gesammelt, während in der Nähe der Quelle, wo vermöge des grössern Umfangs das Wasser ruhiger ist, Gyrinus marinus Gyll., seine Kreise zieht, welchem jedoch der grossen Tiefe wegen nur selten beizukommen ist.

Die Heterocerus-Arten, den grössern parallelus Geb., sowie den kleinern femoralis Kies., haben wir unmittelbar am Ufer in nassem Boden zu suchen, wo sie aus ihren Löchern 
hervorkriechen, sobald sie Geräusch hören; in den ersten Tagen des Frühjahrs sind sie überall im Fluge zu fangen. $\mathrm{Zu}$ dieser Zeit von April bis Anfang Juni kommen auf dem rechten Ufer unter Steinen und Erdspalten die Pogonen, wie luridipennis Germ., iridipennis Nicol. und balophilus Nicol. war. Letzterer ist bis zum Herbste anzutreffen und zu den hier gemeinsten Käfern zu zählen.

Die Salzkäfer haben wir nur, da sie gute Tauschobjecte abgeben, in Menge gesammelt, und nehmen nur noch von den hier vorkommenden folgende mit nach Hause. Von der Distel den Larinus Jaceae, von dem Labkraut Agelactica hallensis, ausserdem noch Cryptophagus pilosus Gyll., Agonum marginatum, Meloë proscarabaeus L., - violaceus M., Carabus cancellatus Ill, auratus L. und Platysma pioimana Duft.

Mit einer Anzahl stearingetränkter Papierblätter versehen, worin wir die Algen einstweilen aufbewahren, um sie zu Hause bei $250-300$ facher Vergrösserung zu untersuchen, beginnen wir nun die Excursion zum Einsammeln der Algen und Bacillarien. Ein Mikroskop führen wir nicht bei uns, das Gefühl muss daher das seinige thun, um eine Partie von der anderen zu unterscheiden. So fühlen sich die Enteremorphen häutig, die Cladophoren rauh und trocken, die Melosiren schleimig, andere wie die Vaucherien seidenartig an, und wir fangen mit dem Einsammeln bei der Quelle an, da hier die interessantesten anzutreffen sind.

Rund um die Quelle herum, mehre Fuss über dem Niveau auf sumpfiger Unterlage treffen wir auf die Vaucheria clavata Ag., einen dunkelgrünen filzartigen Ueberzug bildend, der im Monat August Sporen ansetzt; unmittelbar in der Quelle selbst, $1-2^{\prime}$ unter der Oberfläche und in dem daranstossenden Gerinne die Spirulina solitaris Ktz. Die Fäden der Spirulina sind zu zusammengefilzten Massen vereinigt, und überziehen die ganzen Flächen des Mauerwerkes. Hin und wieder trifft man sie auch völlig rein auf der Oberfläche des Wassers zwischen andern Algen an, und sind sie leicht durch ihre spangrüne Farbe kenntlich, wahrscheinlich durch 
die sich entwickelnde Luft losgerissen. Ihre Form lässt sich mit einem Korkzieher vergleichen, nur vielleicht 500 mal kleiner, und sind sie, so lange sie leben, bei Tag und bei Nacht, Sommer und Winter (die Temperatur ist constant), in fortwährenden Oscillationen begriffen. Die Bewegung besteht in fortwährenden Kreispendelschwingungen, dabei noch vorwärts und rückwärts. Der Durchmesser der Alge ist $1 / 500$ Millimeter bei einer Länge von nur wenigen Windungen bis zu $1 \frac{1}{3}$ Millimeter und dauert bei grössern Exemplaren eine Kreispendelschwingung ohngefähr 5 Secun. den, kleinere rücken unter Drehung nur vor und gehen wieder zurück. Da man linke und rechte Spiralen unterscheidet, 80 geht die Drehung stets nach der Seite, wo die Fadenspitze sich in das Wasser einschrauben kann, die Spitze sonach nicht nachgezogen wird. Häufig beobachtet man doppelt gewundene Fäden. Diese Doppelspiralen sind einfach durch Ineinanderdrehung entstanden und keine besondere Art, indem ich solche beobachtet, die oben ein Oehr bildeten. In süssem Wasser lässt sie sich einige Zeit aufbewahren, in der Soole dagegen wird sie nach kurzer Zeit zerstört, indem sich Schwefelwasserstoffgas entwickelt.

Der Eindruck, den diese Alge auf den Beobachter macht, ist ein im höchsten Grade überraschender, durch das Leben und die stete Bewegung hervorgebracht. In seinem ganzen Laufe ist das Wasser mit dichten Watten der verschiedenartigsten Algen bedeckt, welche Sommer und Winter über wuchern, daher sie, um die Stauung oberhalb der Brücke zu verhindern, von Zeit zu Zeit herausgezogen werden müssen.

Reich vertreten sind die Ulvaceen und zwar Enteromorpha intestinalis L., b) capillaris Rab., c) tubulosa $R$, salina Ktz., b) ramosa A. Ferner Lyngbya salina Ktz. mit Oscillaria maxima Ktz. und major A. Während Chthonoblastus salinus mit Schizosiphon salinus Ktz. mehr auf dem rechten Ufer auf sterilen Stellen vorkommt, füllt Rhizoclonium salinum Ktz., die seichten ausgetretenen Stellen am linken Ufer in ungeheuren Massen aus. Auf den Steinen im Bache neben fuss- 
langen Melosiren die Dasyactis salina Ktz. mit Lyngbya salina und pannosca Ktz.; Schizosiphon salinus Ktz. dagegen überzieht auch hier die Brettereinfassung als krustenartiger Ceberzug. Die Cladophoren wie flavida Ktz., crispata und brachystelecha Rb., sind weiter unten in der Nähe der Unstrut aufzufinden, wo die Einfassung des Gerinnes mit diesen Algen bewachsen ist. Eine Vaucheria, zu clavata gehörend, doch steril, daher nicht genau zu bestimmen, wächst auf dem Grunde der Soole, dicht besetzt mit den verschiedensten Bacillarien, vorzüglich Synedra-Arten.

Die Diatomeen sind überaus reich vertreten, und einzelne Algen wie Rhizoclonium etc. sind förmlich incrustirt. Durch die massenhafte Verbreitung der Melosiren wird das Wasser oft braun gefärbt. Folgende Arten habe ich aufgefunden:

Melosira salina Ktz., Synedra Unna Ehb., S. subtilis Ktz., S. tenuis Ktz., S. saxonica Ktz., Amphiprosa alata Ktz., Diatoma tenue Ktz., Cocconeis salina R., Achnanthes subsessilis Ktz., A. brevipes Ag., Pleurosigma angulata R., Fragilaria Lyngb.? Amphora affinis Ktz., A. coffeaeformis Ktz., Surirella striatula Turp., Epithemia Westermanni Ktz., Stauroneis Phönicenteron Ehb., Mastogloia Danseii Thu., Cyclotella? Ktz., Bacillaria paradoxa Gmel., Campylodiscus costatus Sm., Frustulia salina Ehb., sowie einige mir unbekannte Formen, den Gattungen Pleurosigma, Nitzschia und Navicula angehörend.

Da sie überall vorkommen, genügt es, eine Partie Algen auszudrücken, das trübe Wasser zu sammeln und durch $\mathrm{Ab}$ setzenlassen zu sondern, wodurch man sie ziemlich rein erhält. Behufs Darstellung mikroskopischer Objecte werden dieselben mit Chlorwasserstoffsäure, der man einige Tropfen Salpetersäure zugesetzt hat, einmal aufgekocht. Man lässt dann absetzen, süsst gut aus, und bringt sic dann auf Gläser, die man entweder als trockene Objecte braucht, oder mit einem Tropfen Balsam erwärmt und verschliesst.' Nur Pleurosigma muss trocken aufbewahrt werden, da sic leicht zu durchsichtig wird. 
Noch muss ich bemerken, dass Mastogloia und die Amphoren sich mehr in der Spirulina aufhalten, während Spithemia mehr im Soolschlamm gefunden wird. Die Spirulina wird direct der Einwirkung der Säure ausgesetzt, von welcher sie leicht zersetzt wird, unter Zurücklassung der reinen Kieselpanzer. Alle diese Bacillarien zeigen bei starker Vergrösserung feine Liniensysteme, und können so füglich als Probeobjecte bei Prüfung der Mikroskope benutzt werden. Lässt man bei tiefem Stande der Sonne directes Sonnenlicht bei schiefer Spiegelstellung seitwärts auf Pleurosigma angulatum fallen, so treten bereits bei 300facher Vergrösserung alle drei Liniensysteme gleichzeitig hervor.

So beschliesse ich denn meine Wanderung in dem Glauben, dass es wohl wenige Orte geben mag, wo dem Sammler auf so kleinem Raum von Erde und Wasser eine so reiche Beute zu Theil werde.

\section{Empfangsanzeige.}

Von Herrn Dr. Ernst Hallier, Professor zu Jena, ist ein gedrucktes Sendschreiben an deutsche und auswärtige Gelehrte erschienen, enthaltend: Rechtfertigung gegen die Angriffe des Herrn Professor Dr. de Bary. Der Unterzeichnete erhielt ein Exemplar desselben vom Hrn. Verfasser und hat solches der Vereinsbibliothek einverleibt.

H. $L$. 